\title{
Analytic results for the percolation transitions of the enhanced binary tree
}

\author{
Petter Minnhagen* and Seung Ki Baek \\ Department of Physics, Umeå University, 90187 Umeå, Sweden
}

\begin{abstract}
Percolation for a planar lattice has a single percolation threshold, whereas percolation for a negatively curved lattice displays two separate thresholds. The enhanced binary tree (EBT) can be viewed as a prototype model displaying two separate percolation thresholds. We present an analytic result for the EBT model which gives two critical percolation threshold probabilities, $p_{c 1}=\frac{1}{2} \sqrt{13}-\frac{3}{2}$ and $p_{c 2}=1 / 2$, and yields a size-scaling exponent $\Phi=\ln \left[\frac{p(1+p)}{1-p(1-p)}\right] / \ln 2$. It is inferred that the two threshold values give exact upper limits and that $p_{c 1}$ is furthermore exact. In addition, we argue that $p_{c 2}$ is also exact. The physics of the model and the results are described within the midpoint-percolation concept: Monte Carlo simulations are presented for the number of boundary points which are reached from the midpoint, and the results are compared to the number of routes from the midpoint to the boundary given by the analytic solution. These comparisons provide a more precise physical picture of what happens at the transitions. Finally, the results are compared to related works, in particular, the Cayley tree and Monte Carlo results for hyperbolic lattices as well as earlier results for the EBT model. It disproves a conjecture that the EBT has an exact relation to the thresholds of its dual lattice.

PACS numbers: 64.60.ah,05.70.Jk
\end{abstract}

${ }^{*}$ Corresponding author, E-mail: Petter.Minnhagen@physics.umu.se 

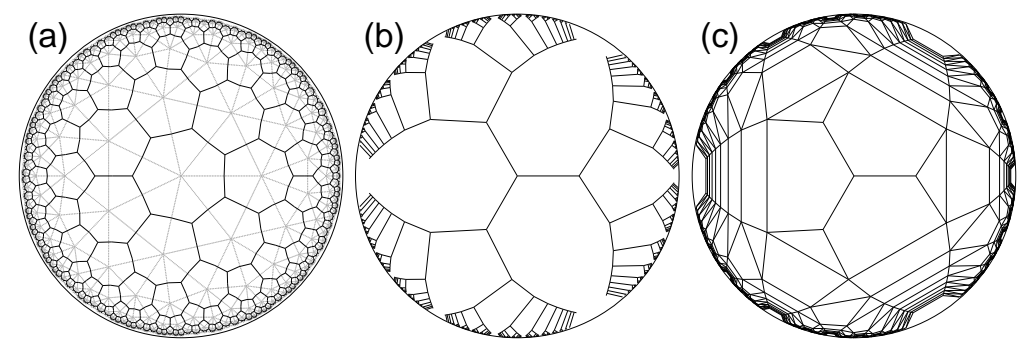

FIG. 1: Hyperbolic lattice structures drawn on the Poincaré disk. (a) The solid lines show a heptagonal lattice, $\{7,3\}$, and the dotted lines show its dual, $\{3,7\}$. (b) A tree structure, represented as $\{\infty, 3\}$, up to the maximum level $L=10$. (c) The enhanced tree obtained from (b) by connecting the tree branches.

\section{INTRODUCTION}

The percolation transition is one of the main examples of a critical phenomena [1, 2], and there have been extensive studies on lattices in Euclidean space, in particular, various types of planar lattices. An interesting and somewhat less studied aspect of the percolation phenomena is that, while a planar lattice possesses a single percolation threshold, a hyperbolic lattice with a constant negative curvature has two different thresholds [3]. Figure 1(a) gives an example of a hyperbolic lattice with Schläfli symbol $\{7,3\}$ as well as its dual, $\{3,7\}$, where $\{m, n\}$ means that the number of regular $m$-gons meeting at each vertex inside the structure is $n$. One characteristic feature of negative curved lattices is that the number of boundary points is a finite fraction of the total number of lattice points. The fact that the number of surface points is a finite fraction is intimately connected to the existence of two thresholds: the probability of reaching one of the boundary point from the midpoint becomes finite at occupation probability $p=p_{c 1}$ in the infinite-size limit, whereas the probability to reach a finite fraction of the boundary points becomes finite at $p_{c 2}$. This midpoint-percolation description offers a conceptually simple way of understanding the physics at the two thresholds. The hyperbolic lattices have, in comparison with the planar lattices, two complications: one is that there are few known exact results for the actual values of the thresholds and for the critical properties associated with them. The other is that, since the number of lattice points grows rapidly with the distance from the midpoint, it is hard to obtain very precise results numerically by using standard size scaling in conjunction with Monte Carlo techniques. The enhanced binary tree (EBT) model alleviates these difficulties to some extent. 
(a)

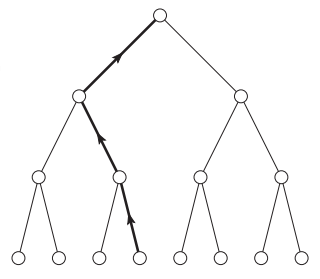

(b)

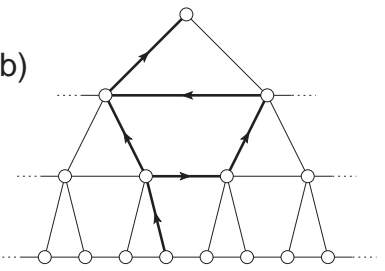

(c)

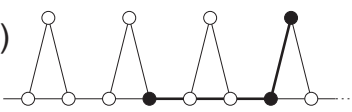

FIG. 2: Schematic representations of (a) the binary tree and (b) EBT. Arrows show examples of routes from the surface to the midpoint. In panel (a), there is only one possible route from a surface point to the top, whereas in panel (b), there are several because the tree branches are connected. (c) A horizontal layer with a rightward tagged route. The three black dots denote the first point of the route on level $l$, the last point of route on level $l$, and the first point of the route on level $l-1$, respectively.

The EBT was introduced in the present context by Nogawa and Hasegawa [4] as a simplified model for a negatively curved lattice. Like the usual Cayley tree, it retains the key feature that the boundary points are a finite fraction of the total number of lattice points. However, the EBT model has an advantage over the usual hyperbolic lattices because its smaller fraction of boundary points, which makes it easier to obtain numerical results for larger sizes. Even so, recent conflicting numerical results for the EBT model reflect the difficulty inherent in obtaining reliable numerical results for hyperbolic lattices [5, 6]. Hence it would be useful to have exact estimates for a prototype model, which can be used as a benchmark for the various numerical techniques (see Refs. [7-14] for some existing exact threshold calculations). It is then fortunate that, as shown here, the EBT model itself possesses analytically accessible results.

The EBT structure is obtained from the usual binary tree by adding horizontal cross links together with a periodic boundary in the horizontal direction, as shown in Figs. 1(c) and 2(b). As is apparent by comparing the hyperbolic lattice in Fig. 1(a) with the EBT in Fig. 1(c), they have similar structures: the essential shared properties are first that the number of lattice points on the surface, $B$, is a finite fraction of the total number of lattice points, $\Omega$, in the limit $\Omega \rightarrow \infty$, and second that there are horizontal paths leading all the way around the lattices. Comparing the binary tree and the EBT in Figs. 2(a) and 2(b) shows that the crucial difference is that the distinct branches for the binary tree are horizontally linked together in the EBT. 


\section{ANALYTIC RESULT}

The method used to find solutions for the EBT model can be illustrated using the binary tree. We pick an arbitrary point on the surface and ask how many routes there are which connect this particular point to the midpoint. For the binary tree the solution is trivial: as seen from Fig. 2(a), there is only one possible route. But in order to reach the top, all the links along this route have to be in place. The chance for a link to be in place is given by the occupation probability $0 \leq p \leq 1$. If the number of steps needed to reach the top is $L$, then the probability to get to the top is $P=p^{L}$, which obviously goes to zero for $L \rightarrow \infty$ unless $p=1$. So, if one picks an arbitrary surface point, there is for $p=1$ a finite probability for the existence of a connected route to the midpoint. This means that all the surface points have a finite probability to be connected to the midpoint. Hence at $p_{c 2}=1$, a finite fraction of the surface point is connected to the midpoint (in this trivial case, this fraction is 1). The number of surface points is $B=2^{L}$, and one can alternatively ask what is the chance for finding a connection from the midpoint to just one of the surface points. This is just $P \cdot B=p^{L} 2^{L}=(2 p)^{L}$, which becomes finite for $p=1 / 2$ and defines the first percolation threshold $p_{c 1}=1 / 2$. Thus the binary tree possesses two separate thresholds: the lower one $p_{c 1}$ defined as the first appearance of a connection from the midpoint to the surface and the second one, $p_{c 2}$, defined as the first appearance of midpoint to surface connections which reaches a finite fraction of the surface points.

We apply the same method to obtain the thresholds for the EBT case: two possible routes from a surface point to the midpoint are drawn in Fig. 2(b). The difference with the binary tree is that a route for the EBT can jump between the various tree branches. Thus there are now many possible routes and the chance that one of them goes all the way to the top for a given $p$ increases. As a consequence, the threshold $p_{c 2}$ decreases to a value smaller than 1 .

We first make a classification of possible routes to the midpoint from the chosen starting point on the surface. A route classification is defined as an ordered sequence of lattice points, route-markers, given by $\left(l, d_{l}, s_{l}\right)$ : if we start from a route-marker point at distance $l$ from the midpoint then the next route-marker point is identified by $d_{l}= \pm 1$, which means that the next is left or right along the horizontal level $l$, together with $s_{l}$ which denotes the distance in lattice points to the next route-marker. At this route marker, the route changes level so that the following route-marker is the closest lattice point on level $l-1$, which means 
that this point is the first point on the route on level $l-1$. From this first point on $l-1$, we then identify the next two route-markers by $\left(l-1, d_{l-1}, s_{l-1}\right)$, which gives the last point on the route on level $l-1$, as well as the first on level $l-2$. The route classification is illustrated in Fig. 2(c). Next we restrict the routes to all the routes which do not contain any backstep, where a backstep is a step which increases the distance to the midpoint. Thus we only consider steps which are either horizontal or upwards in Fig. 2(b). Also note that if $s_{l}=0$, the route goes directly to the closest point on level $l-1$. Thus a complete sequence of $l$ route-marker labels $\left(l, d_{l}, s_{l}\right)$ gives a class of routes from the surface point to the midpoint.

The next step is to consider how we get from the starting point on level $l$ to the end point on level $l-1$ for one specific link configuration. As one moves horizontally on level $l$, one arrives at the possible route-markers in consecutive order. Some of the lattice points are linked to the level above, while others are not. Only the ones which are linked are possible route-markers. These possible route-marker points can be enumerated in consecutive order $s_{l}^{(k)}$ with $k=1,2, \ldots$, so that $\left(l, d_{l}, s_{l}^{(k)}\right)$ enumerates all the possible route classes we can have when starting from the first point on level $l$ going horizontally in the direction $d_{l}$ to the next route marker, which connects to the level $l-1$. Out of these possibly many classes one picks one specific, the one with the shortest horizontal distance $\left(l, d, s_{l}^{(1)}\right)$. By choosing the shortest route-marker distance on every level one obtains a class of tagged routes from the point on the surface to the midpoint. The probability for one such tagged route, denoted by $j$, is $P_{j}^{(\operatorname{tag})}=\prod_{l=0}^{L} P^{(\operatorname{tag})}\left(l, d_{l}, s_{l}^{(1)}\right)$ and the average number of such tagged routes, which reach the midpoint, is $r^{(\operatorname{tag})}=\sum_{j} P_{j}^{(\operatorname{tag})}$. Here $j=1 \ldots 2^{L}$ enumerates the possible tagged routes which correspond to the possible sequences $d(1), d(2), \ldots d(L)$. The point is that $r^{(\operatorname{tag})}$ can be explicitly calculated: starting from the first route-marker on level $l$ in Fig. 2(c), the chance for getting to the closest point on the level $l-1$ above, when moving to the left, is $b(p)=p+(1-p) p^{2}$, because there are two routes leading to the closest point. In the same way, the chance to end at the second closest is $a(p) b(p)$ where $a(p)=p^{2}(1-p)^{2}$, the third $a^{2}(p) b(p)$ and to point number $i$ is $a^{i-1}(p) b(p)$. This also means that the chance of ending at one of the points on the level above, when moving in the left direction starting from a right triangle corner is

$$
Q_{\text {left }}(l)=\sum_{\nu=1}^{N(l-1)} a^{\nu-1}(p) b(p)=b(p) \frac{1-a^{N(l-1)}(p)}{1-a(p)},
$$

where $N(l-1)=2^{l-1}$ is the number of points on the $l-1$ level. In the rightward direction, 
like the route drawn in Fig. 2(c), we first have to move one step to the right with probability $p$ and the result becomes

$$
Q_{\text {right }}(l)=p b(p) \frac{1-a^{N(l-1)}(p)}{1-a(p)} .
$$

The probability for a tagged route with no backsteps is then given by $\prod_{l=1}^{L} Q_{d(l)}(l)$ where $d(l)= \pm 1$ defines a route to the left or right on that particular $l$-level. The average number of such tagged routes is hence

$$
\begin{aligned}
r^{\mathrm{tag}} & =\prod_{l=1}^{L}\left[Q_{\text {left }}(l)+Q_{\text {right }}(l)\right] \\
& =\left[\frac{(1+p) b(p)}{1-a(p)}\right]^{L} \prod_{l=1}^{L}\left[1-a^{N(l-1)}(p)\right]=\left[\frac{p(1+p)}{1-p(1-p)}\right]^{L} \prod_{l=1}^{L}\left[1-p^{2}(1-p)^{2^{l}}\right],
\end{aligned}
$$

which means that

$$
c\left[\frac{p(1+p)}{1-p(1-p)}\right]^{L} \leq r^{\operatorname{tag}} \leq\left[\frac{p(1+p)}{1-p(1-p)}\right]^{L},
$$

where $0<c \leq 1$ is a constant independent of $L$. Thus for $\frac{p(1+p)}{1-p(1-p)}<1$, the average number of tagged routes without backsteps vanishes in the limit $L \rightarrow \infty$. This happens for $p<1 / 2$ which gives the estimate $p_{c 2}=1 / 2$ : at this threshold the probability for having a tagged route from an arbitrary surface point to the midpoint vanishes. In contrast, for $p>1 / 2$ there is a nonzero probability that a finite fraction of the surface points are connected to the midpoint. Since the number of surface points is $B=2^{L}$, this also means that, when $B r^{\text {tag }}$ becomes finite, there is a finite probability that that one of the surface points is connected to the midpoint. This condition is given by $\frac{p(1+p)}{1-p(1-p)}=1 / 2$ and has the solution $p_{c 1}=\frac{1}{2} \sqrt{13}-\frac{3}{2} \approx 0.30278$. Since the calculation is based on a particular subclass of possible routes, these results are either upper bounds or exact.

\section{EXACTNESS ARGUMENT}

The tagged route thresholds values for $p_{c 1}$ and $p_{c 2}$ are by construction upper bounds. The question is then if they also are the exact values. A heuristic argument for the exactness of $p_{c 2}$ goes as follows: at the real $p_{c 2}$ a unique percolating cluster is formed. The formation of such a cluster means that the probability of finding paths, which takes you to the top, suddenly increases. Such a probability increase is, by the tagged-route argument, predicted 

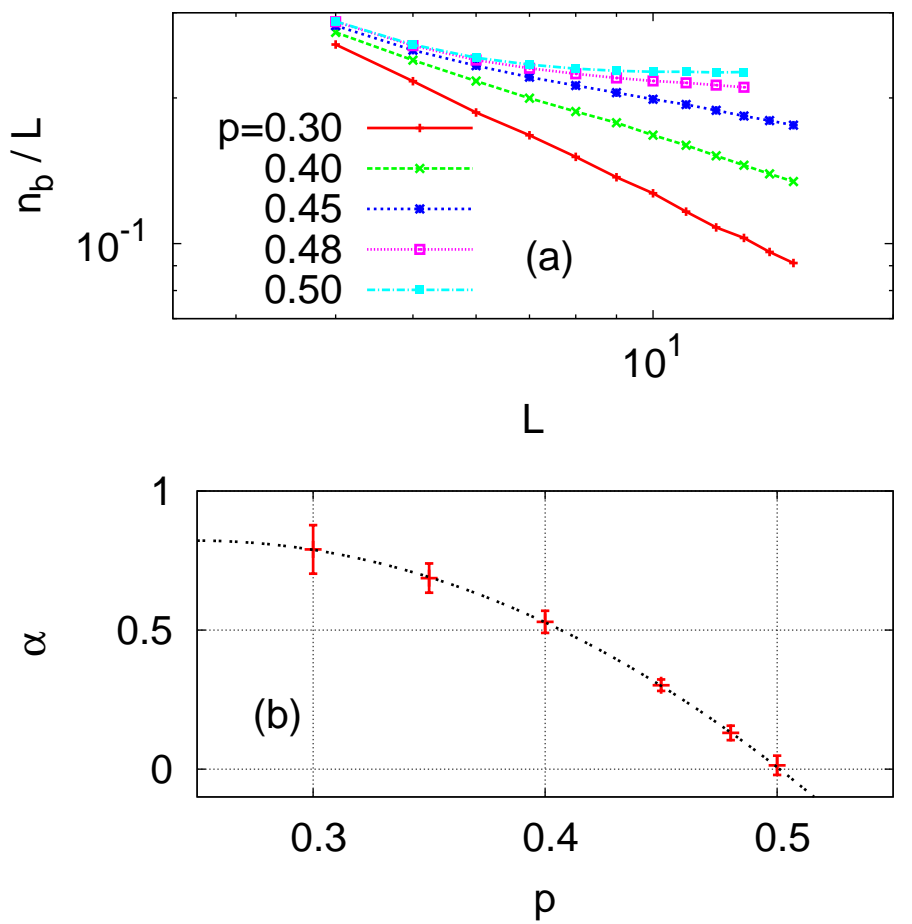

FIG. 3: (Color online) The minimum number of backsteps $n_{b}$ for a nonzero-backstep path which connects from bottom to the top. (a) The data is plotted as $n_{b} / L$ against $L$ in a log-log plot for a sequence of fixed $p$ values. For smaller $p$, the data shows that $n_{b} / L$ vanishes as a power law $n_{b} / L \propto L^{-\alpha}$ with increasing $L$. However, for $p \approx 0.5$, it instead goes to a constant. This change of behavior signals a phase transition. (b) The numerically determined power-law index $\alpha$ plotted as a function of $p$. The dotted line presents a second-order polynomial fit for eyes guidance. Extrapolation for the data points suggests that $\alpha$ vanishes at $p=0.50(1)$.

to happen at $p=0.5$. This suggests that the tagged-route prediction $p_{c 2} \leq 0.5$ can be sharpened to $p_{c 2}=0.5$.

In order to further probe the exactness, we numerically calculate the minimum number of backsteps, $n_{b}$, needed for a nonzero-backstep path to go from the bottom to the top. Figure 3(a) shows that $n_{b} / L$ goes to zero in the limit $L \rightarrow \infty$ in the region below $p=0.5$ and furthermore that this size scaling is a power law $n_{b} / L \propto L^{-\alpha}$. Figure 3(b) shows that the power-law index decreases with increasing $p$ and vanishes at a critical value $p_{c 2} \approx 0.5$. Since this vanishing slope is clearly in itself a critical behavior, it is plausible that the slope vanishes at the exact threshold $p_{c 2} \leq 0.5$. Again the formation of a single percolating cluster at $p_{c 2}$ is likely to be associated with a sudden change of the properties of the routes which 
take you from the bottom to the top. Since a sudden change in the number of backsteps is just such a property, this suggests that $\alpha$ vanishes precisely at $p_{c 2}=0.5$. The numerical results are consistent with vanishing at $p=0.5$, but our numerical precision is not high enough to rule out that it could also vanish somewhere in the region $0.49<p<0.5$.

The fact that the minimum number of backsteps per level vanishes at least below $p=0.49$ makes it possible to deduce that the tagged-route value for the lower threshold $p_{c 1}=\frac{1}{2} \sqrt{13}-$ $\frac{3}{2} \approx 0.30278$ is exact. The reason is that a typical path from the bottom to the top will always contain at least one section where the distance between two backsteps along the path is proportional to $L$. This means that a typical path will always contain sections without backsteps which connects levels separated with a distance which goes to infinity in the limit $L \rightarrow \infty$. The tagged-route argument predicts that such a path exists (does not exist) with a finite probability for $p$ larger (smaller) than $p_{c 1}=\frac{1}{2} \sqrt{13}-\frac{3}{2}$. The same argument tells you that $p_{c 2}$ cannot be lower than the $p$ value where $\alpha$ vanishes, which according to our numerical extrapolation in Fig. B(b) gives $0.49<p_{c 2} \leq 0.5$. Note that the upper limit in this estimate is 0.5 because the tagged-route argument by itself gives an upper bound.

To sum up: we conclude that $p_{c 1}=\frac{1}{2} \sqrt{13}-\frac{3}{2}$ is the exact value and that $0.49<p_{c 2} \leq 0.5$. Furthermore, we conjecture that $p_{c 2}=0.5$ is likewise exact based on plausibility arguments.

\section{COMPARISONS}

Figure 4 gives a comparison between the number of tagged routes connecting the midpoint with the surface, $B r^{\text {tag }}$ given by Eq. (3) , and $b$, the number of surface points connected to the midpoint: Fig. 4(a) shows the number of tagged routes as a function of $p$ for various sizes $L$. The curves for the different sizes cross at the critical value $p_{c 1}=\frac{1}{2} \sqrt{13}-\frac{3}{2} \approx 0.3028$, whereas Fig. 4(b) shows the numbers of surface points which are connected to the midpoint, $b$. These values are obtained from numerical simulations as described in Refs. [3] and [5]. These curves also cross at $p_{c 1}$ and the independent numerical estimate in Ref. [4], $p_{c 1}=0.304(1)$, is also in excellent agreement with this result. Note that $r^{\text {tag }}$ and $b$ are two distinct quantities although closely related. They would be identical if the connection between a surface point

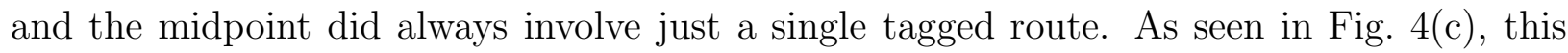
is true to good approximation in the region $0.3<p<0.4$. For small $p$, there is a tiny deviation because a single tagged route has a small but finite chance of connecting more 

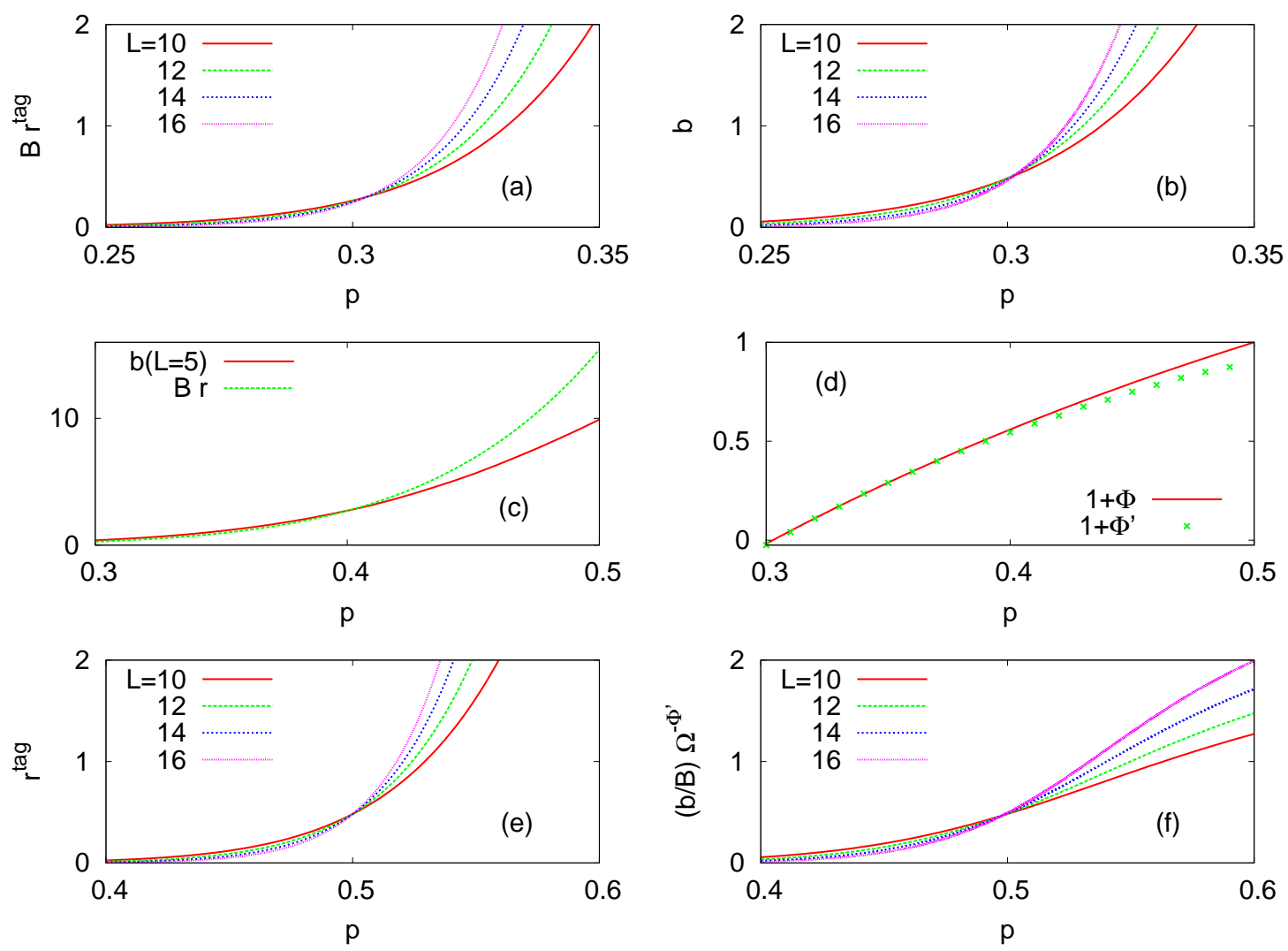

FIG. 4: (Color online) Comparisons between $r^{\text {tag }}$, the average number of tagged routes from an arbitrary surface point to the midpoint, and, $b$, the number of surface points connected to the midpoint. $r^{\text {tag }}$ is given by the analytical solution and $b$ is obtained from numerical simulations. (a) The number of tagged routes from the surface to the midpoint, $B r^{\text {tag }}$ for various sizes $L$. All curves cross in a single point at $p_{c 1} \approx 0.303$. (b) The same plot for $b$. Note the strong resemblance and the single crossing point at $p \approx 0.303(1)$. The panel (c) illustrates the close connection between $B r^{\text {tag }}$ and $b$. For $p \leq 0.4$ the number of routes and the number of connections are almost the same (the number of routes is slightly smaller as explained in the text). However for $p \geq 0.4$, the number of routes exceeds the number of connected surface points. The panel (d) illustrates this connection further by comparing the size-scaling exponent $1+\Phi$ obtained analytically for $r^{\text {tag }}$ with the size scaling for $b$ obtained from (b). The agreement is striking for $p \leq 0.4$. (e) $r^{\text {tag }}$ for various sizes with the crossing point at $p_{c 2}=1 / 2$. (f) The corresponding crossing point for $b / B$ obtained numerically. The crossing point is close to 0.50 , but the size-scaling exponent $\Phi$ is different. For $r^{\text {tag }}$ the value is $\Phi\left(p_{c 2}\right)=0$ but for $b$ it is $\Phi^{\prime}\left(p_{c 2}\right) \approx-0.11$, as explained in the text. 
than one surface point to the midpoint. Thus if a surface point and a midpoint is always connected by precisely one route, then the total number of routes is slightly smaller than the number of connected surface points. On the other hand, for larger $p$, many routes can be connected to the same surface point, so that the number of routes instead exceeds the number of connected surface points. This effects becomes noticeable for $p>0.4$. Figure 4 (c) demonstrates the strong connection between the tagged routes and the connections between the midpoint and the surface. This correspondence is further enforced by comparing the size dependence of $r^{\mathrm{tag}}$ and $b / B$. For $r^{\mathrm{tag}}$ the size dependence follows from Eq. (44) from which one obtains $r^{\text {tag }} \sim \Omega^{\Phi}$, where $\Omega \sim 2^{L}$ is the size of the system and $\left.\Phi=\ln \left[\frac{p(1+p)}{1-p(1-p)}\right)\right] / \ln 2$. The exponent $\Phi$ is plotted in Fig. 4(b) and is compared to the corresponding exponent for $b / B \sim \Omega^{\Phi^{\prime}}$ obtained numerically from simulations [3, 5]. As seen $\Phi \approx \Phi^{\prime}$ for $p<0.4$, which again shows that in this interval the number of connected surface points is closely the same as the number of tagged routes. However, as noticed above, for larger values of $p$, the number of routes exceeds the number of connected surface points. This has interesting consequences at the second threshold $p_{c 2}$ : in Fig. 4(e), the number of tagged routes per surface point is plotted and the curves for the various sizes crosses precisely at $p_{c 2}=0.5$. This means that, at this threshold, the size-scaling exponent for the tagged routes is $\Phi=0$ whereas the numerically determined size-scaling exponent for the fraction of attached surface points, $b / B$ is $\Phi^{\prime} \approx-0.11$, as shown in Fig. 4(e). This means that, at $p_{c 2}$, the number of tagged routes which are attached to the same surface point decreases with size as a power law with the exponent $\Phi^{\prime} \approx-0.11$. One also notes that the threshold value determined numerically from $b / B$ is consistent with the exact value $p_{c 2}=1 / 2$, but with a larger uncertainty than the numerical determination of $p_{c 1}$.

One question is whether or not the EBT and its dual lattice are related in such a way that the lower threshold $p_{c 1}^{(\mathrm{dual})}$ for the dual lattice and the upper threshold for the EBT $p_{c 2}$, sum to $p_{c 1}^{\text {(dual) }}+p_{c 2}=1$, as was conjectured by Nogawa and Hasegawa [4]. Just as for the EBT, the lower threshold for the dual lattice can be numerically determined to good precision and is given by $p_{c 1}^{\text {(dual) }}=0.436(1)[4]$. The determination of $p_{c 2} \leq 1 / 2$ in this work shows that in fact $p_{c 1}^{\text {(dual) }}+p_{c 2} \leq 0.936<1$, and that consequently the conjectured exact relation is not valid. This property is shared with the hyperbolic lattices. For example, $p_{c 2}$ is for the $\{7,3\}$ lattice in Fig. 1(a) numerically determined as $p_{c 2} \approx 0.72$ whereas the lower threshold for the dual lattice $\{3,7\}$ is approximately $p_{c 1}^{(\text {dual })} \approx 0.20[3]$. This gives $p_{c 1}^{(\text {dual })}+p_{c 2} \approx 0.92$ and 
illustrates the strong resemblance between the EBT model and the hyperbolic lattices.

\section{SUMMARY}

The values of the two percolation thresholds for the EBT model were inferred using a two-step method: the first step involved an exact calculation of a particular class of routes from the surface to the midpoint. The second step involved a numerical calculation of the minimum number of backsteps for nonzero-backstep routes connecting a surface point to the midpoint: when this number increases slower than $L$, the tagged-route probability determines the percolation. This made it possible to infer the exact value of $p_{c 1}$. Arguments were presented which, in our opinion, give strong evidence that $p_{c 2}$ is also given exactly by the tagged-route probability. One might suspect that there are lattices for which complete analytical solutions are difficult to find, where the tagged-route probability determines the percolation threshold. For example, the exact results for the triangular, quadratic, and honeycomb lattices are readily reproduced.

Hyperbolic lattices in general have two separate percolation thresholds and both of them can be identified within the midpoint-percolation concept: at the lower threshold, a finite number of the surface points are reached from the midpoint and at the higher, a finite fraction. The EBT can be viewed as a simplified version of a hyperbolic lattice and it is, to our knowledge, the first lattice of this type with two nontrivial thresholds where at least one has been exactly determined. There are also two trivial cases, i.e., the Cayley tree with $p_{c 1}=1 / 2$ and $p_{c 2}=1$ and the dual to the Cayley tree which has $p_{c 1}=0$ and $p_{c 2}=1 / 2$. In addition, there are related models which also possess two percolation thresholds [13-15]. In Ref. [14], the two thresholds for a scale-free hierarchical network were exactly obtained and found to be $p_{c 1}=0$ and $p_{c 2}=5 / 32$, which seems superficially somewhat reminiscent of the dual to the Cayley tree in the sense that there is no nonpercolating phase for any nonzero $p$. Likewise, two percolation thresholds were found for a certain Hanoi network in Ref. [13] and determined to high precision. In this case, both thresholds are nonzero just as for the EBT. However, the relation between the scale-free hierarchical network and the Hanoi network, on the one hand, and the percolation transitions for a hyperbolic lattice, on the other, is an interesting question which remains to be elucidated.

Apart from being a good proxy of a hyperbolic lattice, the EBT has interesting intrinsic 
properties as illustrated by the size scaling and the close relation between the tagged routes and the surface-to-midpoint connections. Consequently, it might turn out to be a useful model for future studies of percolation on hyperbolic lattices, as well as for percolation in general.

\section{Acknowledgments}

We acknowledge the support from the Swedish Research Council with the Grant No. 6212002-4135. This research was conducted using the resources of High Performance Computing Center North (HPC2N).

[1] D. Stauffer and A. Aharony, Introduction to Percolation Theory, 2nd ed. (Taylor \& Francis, London, 2003).

[2] K. Christensen and N. R. Moloney, Complexity and Criticality (Imperial College Press, London, 2005).

[3] S. K. Baek, P. Minnhagen, and B. J. Kim, Phys. Rev. E 79, 011124 (2009).

[4] T. Nogawa and T. Hasegawa, J. Phys. A 42, 145001 (2009).

[5] S. K. Baek, P. Minnhagen, and B. J. Kim, J. Phys. A 42, 478001 (2009).

[6] T. Nogawa and T. Hasegawa, J. Phys. A 42, 478002 (2009).

[7] M. F. Sykes and J. W. Essam, Phys. Rev. Lett. 10, 3 (1963).

[8] H. Kesten, Commun. Math. Phys. 74, 41 (1980).

[9] J. C. Wierman, J. Phys. A 17, 1525 (1984).

[10] C. R. Scullard, Phys. Rev. E 73, 016107 (2006).

[11] R. M. Ziff, Phys. Rev. E 73, 016134 (2006).

[12] R. M. Ziff and C. R. Scullard, J. Phys. A 39, 15083 (2006).

[13] S. Boettcher, J. L. Cook, and R. M. Ziff, Phys. Rev. E 80, 041115 (2009).

[14] A. N. Berker, M. Hinczewski, and R. R. Netz, Phys. Rev. E 80, 041118 (2009).

[15] T. Hasegawa and K. Nemoto, Phys. Rev. E 81, 051105 (2010). 